\title{
Tendência histórica de preços pagos ao produtor na agricultura de grãos do Rio Grande do Sul, Brasil
}

\author{
Price tendency received by grain agricultural farmers in Rio Grande do Sul, Brazil
}

\author{
Renato Santos de Souza' João Garibaldi Almeida VianaII
}

\section{RESUMO}

O presente trabalho analisa a evolução histórica dos preços pagos ao produtor pelos principais produtos da agricultura do Rio Grande do Sul nas últimas três décadas, dando especial atenção às tendências ocorridas a partir da década de 90 e, principalmente, após a estabilização monetária obtida com o Plano Real de 1994. A metodologia utilizada foi a de análise de séries temporais, realizada com base no histórico de preços médios mensais pagos ao produtor, coletados pela Emater/RS, abrangendo o período de janeiro de 1973 a dezembro de 2005. A análise permitiu concluir que todos os produtos pesquisados (arroz, feijão, milho, soja e trigo) apresentaram uma desvalorização significativa em seus preços reais entre os anos de 1973 e 2005, e que a década de 80 foi o período de maior declínio de preços. Além disso, constatou-se que o período após o Plano Real foi marcado pela inexistência de tendência de preços para arroz, trigo e milho, sobretudo pela forma errática com que os mesmos variaram, e pela leve tendência de elevação de preços da soja e de declínio de preços do feijão.

Palavras-chave: preços agrícolas, agricultura gaúcha, séries temporais.

\section{ABSTRACT}

This research examines the historical evolution of prices paid to farmers for the most important agricultural products in Rio Grande do Sul for the last three decades, by giving special attention to the changes in prices that occurred since the nineties, and mainly after the monetary stabilization obtained with the "Plano Real" in 1994. The methodology used was the times series, based on longitudinal data collected by Emater/RS regarding the average monthly prices paid to farmers, from January 1973 to December 2005. The results indicated that all studied products (rice, beans, corn, soy, and wheat) had a significant decline in their real price values between the years of 1973 and 2005, and the biggest decline occured during the eighties. Besides, it was not identified a clear price trend for rice, wheat and corn in the period after the "Plano Real" due to the random pattern in price variation for these products. In this period, there was also a slight positive trend in the prices of soy and a decline in the prices of beans.

Key words: agricultural prices, livestock market, times series.

\section{INTRODUÇÃO}

Embora seja comum o desenvolvimento tecnológico vir acompanhado por aumento na oferta de produtos e por redução nos preços agrícolas, e que este comportamento, por implicar aumento do poder de compra dos salários, tenha sido uma das principais funções da agricultura na maioria dos países desenvolvidos (VEIGA, 1990), não há consenso, para o caso brasileiro, sobre o desempenho do setor neste aspecto.

Algumas pesquisas que trabalharam com índices nacionais de preços pagos ao produtor agropecuário e de custo da alimentação dão conta de que, no agregado, estes subiram mais do que o conjunto de preços da economia em boa parte do período posterior à década de 70 , contradizendo, simultaneamente, teorias e experiências internacionais de desenvolvimento agrícola, bem como crenças estabelecidas entre os agentes econômicos rurais.

Este é o caso, por exemplo, das pesquisas de VEIGA (1990) e CARMO (1996), que concluíram,

IDepartamento de Educação Agrícola e Extensão Rural, Universidade Federal de Santa Maria (UFSM), Prédio 44, Faixa de Camobi km 09, 97105-900, Santa Maria, RS. E-mail: rssouza@smail.ufsm.br. Autor para correspondência.

${ }^{\text {IIC }}$ urso de Pós-graduação em Extensão Rural, UFSM, Santa Maria, RS, Brasil. 
para o período imediatamente anterior às respectivas publicações, que há décadas o Brasil convivia com uma elevação dos preços reais dos alimentos. $\mathrm{Na}$ pesquisa de Carmo, os resultados davam conta de que tanto os preços dos alimentos como os preços pagos ao produtor haviam apresentado aumentos superiores à inflação nas últimas décadas analisadas na pesquisa. Segundo a autora, os preços da alimentação apresentaram uma tendência crescente face aos preços médios da economia desde 1973, enquanto que o índice de preços recebidos pelos agricultores teria se situado sistematicamente acima da linha referencial do Índice Geral de Preços da economia nas décadas de 70 e 80, indicando pressões altistas sobre o crescimento geral dos preços.

Em estudo mais recente, FARINA \& NUNES (2004) demonstraram que, entre 1990 e 2004, os preços agrícolas aumentaram mais do que os preços industriais e os preços dos alimentos, indicando que, de uma maneira geral, ao contrário do que se acreditava quando era atribuído ao setor primário o papel de "âncora verde” do Plano Real, eles não tiveram contribuição significativa para o controle da inflação.

Contradizendo este conjunto de resultados, o estudo de SOUZA et al. (2006) concluiu que todos os sete produtos pecuários estudados (boi gordo, cordeiro, ovelha, suínos, frango de corte, ovos e leite) apresentaram tendência declinante dos preços reais ao produtor, sobretudo até 1994. O trabalho, porém, também está de acordo com parte das conclusões de FARINA \& NUNES (2004), pois detectou, para aqueles produtos, uma relativa estabilização nos preços após 1994, embora não tenha sido observado aumento nos preços relativos pagos ao produtor, exceto para os produtos da ovinocultura.

Assim, essas referências mostram que ainda são precários os estudos sobre séries históricas de preços agropecuários no Brasil, sobretudo aqueles que segmentem a análise por produto e por região. Não se pode generalizar para todo o setor agropecuário conclusões que, baseadas em dados agregados e genéricos, contrariam ao mesmo tempo o senso comum, a teoria e as evidências internacionais sobre o desenvolvimento agrícola.

O problema de pesquisa que motivou o presente trabalho está em desvendar a contribuição do setor primário brasileiro para a disponibilização de alimentos abundantes e baratos para os consumidores urbanos. Trata-se da continuidade do trabalho já desenvolvido para os preços dos produtos pecuários, cujos resultados encontram-se publicados em SOUZA et al. (2006). De outro lado, diz respeito também às implicações do processo de evolução e modernização da agricultura brasileira sobre os preços agrícolas e, consequentemente, sobre a renda dos produtores.

Dessa forma, esta pesquisa teve por objetivo analisar a evolução histórica dos preços pagos ao produtor pelos principais produtos da agricultura de grãos do Rio Grande do Sul (arroz, feijão milho, soja e trigo) nas últimas três décadas, dando especial atenção às tendências ocorridas a partir da década de 90 e, principalmente, após a estabilização monetária alcançada com o Plano Real de 1994.

Espera-se que o presente artigo, juntamente com o anteriormente citado, contribua para que se tenha uma visão retrospectiva do desempenho da agricultura gaúcha, sem perder de vista a dinâmica produtiva, tecnológica e mercadológica de cada produto.

\section{MATERIAL E MÉTODOS}

O estudo foi realizado com base no histórico de preços médios mensais pagos ao produtor, coletados pela Emater do Rio Grande do Sul e disponibilizados para esta pesquisa por intermédio do Centro Integrado de Ensino, Pesquisa e Extensão Rural - Cieper, convênio da Emater com a Universidade Federal de Santa Maria. Os dados abrangem o período de janeiro de 1973 a dezembro de 2005, por ser o disponível na Emater. Os preços estudados referem-se aos seguintes produtos: arroz, feijão, milho, soja e trigo.

A metodologia utilizada no presente trabalho é idêntica à utilizada em SOUZA et al. (2006), e consistiu, inicialmente, em fazer as correções monetárias nos preços nominais históricos relativas aos diferentes planos econômicos (Plano Cruzado, Plano Verão, Plano Collor e Plano Real), e deflacionar os preços corrigidos de cada mês de forma a atualizá-los para valores reais equivalentes a dezembro de 2005. Para o deflacionamento dos preços nominais, foi utilizado o Índice Geral de Preços (IGP-DI), calculado pela Fundação Getulio Vargas, por ser um índice que reflete tanto preços ao consumidor final como preços no interior das cadeias produtivas e canais de comercialização, classificados como preços no atacado. Para cada um dos produtos analisados, foi também calculado um índice de evolução dos preços reais médios anuais, com base 100 em janeiro de 1973, de forma a permitir comparações entre os movimentos de preços dos diferentes produtos.

Por fim, buscou-se verificar a existência de tendência de preços para os produtos estudados. Em análise de séries temporais, o termo tendência descreve um movimento suave, a longo prazo, dos dados, para cima ou para baixo (STEVENSON, 1981), de forma que a análise de tendência diz respeito à verificação da 
direção do movimento de longo prazo na série temporal (KAZMIER, 1982).

Várias são as formas de se avaliar a existência ou não de tendência em uma série temporal de dados, dentre elas estão o uso da taxa geométrica de crescimento (FINAMORE \& GOMES, 1999) e do coeficiente angular da reta de regressão dos dados (STEVENSON, 1981; KAZMIER, 1982). Em razão da simplicidade de interpretação e da eficiência do segundo método em relação ao objetivo proposto, optou-se por ele.

Sabe-se que uma reta nem sempre é o tipo de função que melhor se ajusta aos dados de séries temporais. Porém, procurar o tipo de função mais ajustada só é relevante quando o objetivo for a previsão, com base na equação de tendência, do comportamento futuro da variável que está em análise. Isto, no entanto, só é viável para aquelas variáveis em que o comportamento futuro é definido de forma inercial e que só dependem de fatores estruturais endógenos, que não é o caso dos preços. Quando o objetivo é apenas verificar a existência ou não de tendência histórica e a direção desta tendência, como no caso do presente trabalho, a reta é uma forma funcional adequada.

Assim, para a análise de tendência dos preços reais pagos ao produtor, foram ajustadas retas de regressão sobre os preços reais para os períodos de 1973-1994, 1995-2005 e 1973-2005, utilizando-se o método dos mínimos quadrados, conforme KARMEL \& POLASEK(1976) eJOHNSTON(1977). Tal regressão, com a qual se estimou a equação abaixo, teve como variável dependente o preço real médio anual desestacionalizado e, como variável independente, o período de tempo (em anos).

$$
\mathrm{PT}=\mathrm{a}+\mathrm{b} . \mathrm{T}
$$

Em que: $\mathrm{PT}=$ Preço de tendência; $a=$ coeficiente linear da reta de tendência; $b=$ coeficiente angular da reta de tendência; $\mathrm{T}=$ Período de tempo (1, $2,3,4,5 \ldots n)$.

Como a regressão foi feita com base no preço anual médio desestacionalizado, foi necessário calcular, para cada mês, o “Índice de Estacionalidade” médio, o que se fez utilizando o método das médias móveis de 13 meses preconizado por KAZMIER (1982). Após isso, os preços mensais foram desestacionalizados para retirar dos mesmos as variações estacionais e de curto prazo, deixando na série temporal apenas as variações cíclicas e de tendência e possibilitando, com isso, que se fizesse a regressão com base nos preços médios anuais, o que é mais conveniente e dá maior consistência à análise de tendência.
A existência ou não de tendência declinante ou ascendente nos preços reais dos diferentes produtos no período analisado foi verificada através do teste de hipóteses t-student, com o qual se avaliou, a um nível de 95\% de significância, se o coeficiente angular "b" da reta de regressão ajustada aos dados era significativamente diferente de zero. Quando o "t calculado" é maior que o "t tabelado", o coeficiente angular "b” é significativamente diferente de zero (e vice-versa), o que caracteriza a existência de tendência, ascendente se "b" for positivo ou declinante se for negativo.

Maiores detalhes sobre a metodologia do trabalho podem ser consultados em SOUZA et al. (2006).

\section{RESULTADOS E DISCUSSÃO}

Os produtos agrícolas pesquisados apresentaram queda significativa de preço real no período analisado, como pode ser observado nas tabelas 1 e 2, com desvalorizações de preço entre 1973 e 2005, de 67,3\% para o arroz, 68,1\% para o feijão, 67,1\% para o milho, $79,9 \%$ para o soja e $76,0 \%$ para o trigo.

Esta desvalorização acentuada tem como causa fundamental a expansão das áreas de lavouras e o incremento de tecnologia no campo, resultando no aumento considerável da produção e produtividade das culturas. O acréscimo de produtividade das principais culturas agrícolas é relatado por BARROS et al. (2001), que ainda destacam a transferência dos ganhos de produtividades para os consumidores, 0 que os torna os principais beneficiados pelas quedas nos preços agrícolas.

Durante a década de 70, os produtos arroz, milho e trigo apresentaram elevação de preço, ao contrário dos produtos feijão e soja. Os preços dos produtos agrícolas caíram fortemente durante toda a década de 80, comportamento este que pode ser explicado pela redução na demanda por alimentos, associada à queda na renda per capita da população, ao crescente desemprego, ao aumento da produtividade total dos fatores, às altas taxas de inflação e aos elevados juros, o que acarretou no aumento da instabilidade dos preços (REZENDE, 2003; GASQUES \& CONCEIÇÃO, 2000).

Cabe ressaltar a queda drástica de preços do trigo durante o final da década de 80 e o início da década de 90, decréscimo este influenciado pela queda gradativa da intervenção governamental no setor a partir de 1986, e que levou à exclusão total dos subsídios ao trigo em 1990 com o decreto do Plano Collor (GRAZIANO DA SILVA, 1998). 
Tabela 1 - Preços reais médios*(R\$) pagos ao produtor, atualizados para dezembro de 2005, dos principais produtos da agricultura de grãos do Rio Grande do Sul.

\begin{tabular}{|c|c|c|c|c|c|}
\hline $\begin{array}{l}\text { Produto } \\
\text { Ano }\end{array}$ & $\begin{array}{c}\text { Arroz } \\
(\mathrm{R} \$ / \mathrm{Sc})\end{array}$ & $\begin{array}{c}\text { Feijão } \\
(\mathrm{R} \$ / \mathrm{Sc})\end{array}$ & $\begin{array}{c}\text { Milho } \\
(\mathrm{R} \$ / \mathrm{Sc})\end{array}$ & $\begin{array}{c}\text { Soja } \\
(\mathrm{R} \$ / \mathrm{Sc})\end{array}$ & $\begin{array}{c}\text { Trigo } \\
\text { (R\$/Sc) }\end{array}$ \\
\hline 1973 & 63,64 & 242,36 & 54,54 & 137,85 & 77,41 \\
\hline 1974 & 73,49 & 228,49 & 60,68 & 109,95 & 92,92 \\
\hline 1975 & 92,60 & 141,08 & 56,23 & 93,26 & 109,98 \\
\hline 1976 & 70,93 & 207,67 & 58,82 & 88,40 & 99,61 \\
\hline 1977 & 57,33 & 234,92 & 47,24 & 92,00 & 86,54 \\
\hline 1978 & 67,10 & 174,21 & 56,63 & 86,44 & 93,72 \\
\hline 1979 & 81,21 & 151,76 & 64,16 & 93,07 & 82,76 \\
\hline 1980 & 83,71 & 313,65 & 61,49 & 80,19 & 70,43 \\
\hline 1981 & 61,96 & 289,85 & 50,19 & 70,26 & 73,21 \\
\hline 1982 & 74,87 & 144,75 & 39,76 & 66,42 & 88,23 \\
\hline 1983 & 68,28 & 145,43 & 58,13 & 87,52 & 74,50 \\
\hline 1984 & 60,84 & 170,86 & 51,84 & 96,50 & 86,14 \\
\hline 1985 & 67,11 & 167,12 & 46,59 & 74,79 & 98,66 \\
\hline 1986 & 66,75 & 173,17 & 49,82 & 66,14 & 99,32 \\
\hline 1987 & 39,96 & 113,82 & 30,16 & 58,47 & 59,75 \\
\hline 1988 & 42,00 & 146,34 & 36,58 & 77,03 & 52,78 \\
\hline 1989 & 37,58 & 172,27 & 32,29 & 53,80 & 38,00 \\
\hline 1990 & 37,77 & 111,96 & 25,28 & 33,10 & 26,89 \\
\hline 1991 & 55,99 & 96,65 & 29,36 & 41,69 & 27,35 \\
\hline 1992 & 37,90 & 85,69 & 23,60 & 43,26 & 31,70 \\
\hline 1993 & 33,47 & 84,03 & 24.58 & 42,37 & 29,05 \\
\hline 1994 & 34,26 & 108,88 & 21,34 & 37,06 & 24,37 \\
\hline 1995 & 27,49 & 86,92 & 17,27 & 27,62 & 22,27 \\
\hline 1996 & 28,56 & 67,69 & 20,84 & 36,40 & 27,27 \\
\hline 1997 & 30,48 & 84,26 & 16,64 & 38,80 & 19,83 \\
\hline 1998 & 38,64 & 127,76 & 18,91 & 30,47 & 20,12 \\
\hline 1999 & 29,41 & 69,04 & 20,17 & 32,31 & 23,09 \\
\hline 2000 & 21,65 & 46,66 & 20,48 & 30,90 & 21,85 \\
\hline 2001 & 25,62 & 86,68 & 14,72 & 35,53 & 23,68 \\
\hline 2002 & 28,73 & 94,85 & 21,58 & 43,49 & 30,36 \\
\hline 2003 & 37,42 & 77,54 & 20,30 & 42,80 & 30,97 \\
\hline 2004 & 33,37 & 69,47 & 19,43 & 41,59 & 24,47 \\
\hline 2005 & 20,81 & 77,27 & 17,94 & 27,70 & 18,55 \\
\hline
\end{tabular}

*Deflacionamento dos preços nominais pelo Índice Geral de Preços (IGP-DI) - FGV.

Fonte: Cálculos dos autores a partir de dados primários da EMATER-RS

Com o advento do Plano Real e as novas medidas econômicas de controle inflacionário, a abertura do mercado e a valorização cambial, cessaram as quedas bruscas de preços, não sendo observado um comportamento estável nos mesmos.

As políticas de importações, principalmente de trigo e arroz, influenciadas pela flutuação do câmbio, tiveram forte influência nas variações de preços destes produtos pós-estabilização econômica no mercado interno, alternando anos de elevação e queda nos preços. Os preços da soja também foram influenciados pela flutuação cambial. No período de desvalorização do real, entre 1999 e 2003, os preços elevaram-se, visto a comercialização deste produto para o mercado externo. A nova valorização do real frente ao dólar, a partir de 2003, trouxe mais um fator para a queda nos preços de todos os produtos analisados, decretando a crise no setor agrícola observada nos últimos anos.

A tabela 3 mostra os resultados da regressão elaborada para fins de análise de tendência. Conforme os dados da tabela, todos os produtos apresentaram acentuada tendência declinante de preços no período 1973-2005, o mesmo ocorrendo no período 1973-1994. Porém, ao analisar o período 1995-2005, posterior à estabilização econômica, observa-se que os produtos

Tabela 2 - Índices de preços médios anuais pagos ao produtor dos principais produtos da agricultura gaúcha (base 100 em 1973).

\begin{tabular}{|c|c|c|c|c|c|}
\hline Produto Ano & Arroz & Feijão & Milho & Soja & Trigo \\
\hline 1973 & 100,00 & 100,00 & 100,00 & 100,00 & 100,00 \\
\hline 1974 & 115,47 & 94,28 & 111,25 & 79,76 & 120,03 \\
\hline 1975 & 145,49 & 58,21 & 103,10 & 67,66 & 142,07 \\
\hline 1976 & 111,45 & 85,69 & 107,84 & 64,13 & 128,68 \\
\hline 1977 & 90,08 & 96,93 & 86,61 & 66,74 & 111,79 \\
\hline 1978 & 105,43 & 71,88 & 103,83 & 62,71 & 121,06 \\
\hline 1979 & 127,60 & 62,62 & 117,63 & 67,52 & 106,91 \\
\hline 1980 & 131,53 & 129,41 & 112,73 & 58,18 & 90,98 \\
\hline 1981 & 97,36 & 119,59 & 92,02 & 50,97 & 94,57 \\
\hline 1982 & 117,64 & 59,73 & 72,89 & 48,19 & 113,98 \\
\hline 1983 & 107,28 & 60,00 & 106,58 & 63,49 & 96,24 \\
\hline 1984 & 95,60 & 70,50 & 95,05 & 70,01 & 111,28 \\
\hline 1985 & 105,45 & 68,96 & 85,41 & 54,26 & 127,45 \\
\hline 1986 & 104,88 & 71,45 & 91,34 & 47,98 & 128,30 \\
\hline 1987 & 62,78 & 46,96 & 55,29 & 42,42 & 77,18 \\
\hline 1988 & 65,99 & 60,38 & 67,08 & 55,88 & 68,19 \\
\hline 1989 & 59,05 & 71,08 & 59,21 & 39,03 & 49,10 \\
\hline 1990 & 59,35 & 46,19 & 46,36 & 24,02 & 34,74 \\
\hline 1991 & 87,97 & 39,88 & 53,83 & 30,24 & 35,33 \\
\hline 1992 & 59,55 & 35,36 & 43,27 & 31,39 & 40,96 \\
\hline 1993 & 52,59 & 34,67 & 45,07 & 30,74 & 37,53 \\
\hline 1994 & 53,82 & 44,93 & 39,14 & 26,88 & 31,48 \\
\hline 1995 & 43,19 & 35,86 & 31,67 & 20,04 & 28,78 \\
\hline 1996 & 44,88 & 27,93 & 38,21 & 26,41 & 35,23 \\
\hline 1997 & 47,89 & 34,77 & 30,52 & 28,15 & 25,62 \\
\hline 1998 & 60,72 & 52,71 & 34,67 & 22,11 & 26,00 \\
\hline 1999 & 46,20 & 28,49 & 36,98 & 23,44 & 29,83 \\
\hline 2000 & 34,01 & 19,25 & 37,55 & 22,42 & 28,23 \\
\hline 2001 & 40,25 & 35,77 & 27,00 & 25,77 & 30,59 \\
\hline 2002 & 45,14 & 39,13 & 39,56 & 31,55 & 39,23 \\
\hline 2003 & 58,80 & 31,99 & 37,22 & 31,05 & 40,01 \\
\hline 2004 & 52,43 & 28,67 & 35,63 & 30,17 & 31,62 \\
\hline 2005 & 32,69 & 31,88 & 32,88 & 20,09 & 23,97 \\
\hline
\end{tabular}

Fonte: Cálculos dos autores a partir de dados primários da EMATER-RS.

Ciência Rural, v.37, n.4, jul-ago, 2007. 
Tabela 3 - Parâmetros “a” e “b” e estatística t-student para a regressão de tendência de diferentes períodos na série temporal de preços reais pagos ao produtor.

\begin{tabular}{|c|c|c|c|}
\hline Período & $1973-2005$ & $1973-1994$ & $1995-2005$ \\
\hline \multicolumn{4}{|l|}{ Produto } \\
\hline “t” tabelado a 95\% de significância & $2,021^{*}$ & 2,080 & 2,262 \\
\hline \multicolumn{4}{|l|}{ Arroz } \\
\hline Parâmetro “a” & 79,274 & 82,019 & 29,997 \\
\hline Parâmetro “b” & $-1,864$ & $-2,140$ & $-0,149$ \\
\hline “t” calculado & 19,838 & 21,183 & 1,005 \\
\hline \multicolumn{4}{|l|}{ Feijão } \\
\hline Parâmetro “a” & 227,316 & 237,189 & 85,950 \\
\hline Parâmetro “b” & $-5,513$ & $-6,569$ & $-1,014$ \\
\hline “t” calculado & 14,676 & 25,830 & 3,168 \\
\hline \multicolumn{4}{|l|}{ Milho } \\
\hline Parâmetro “a” & 61,552 & 64,615 & 18,541 \\
\hline Parâmetro "b" & $-1,597$ & $-1,914$ & 0,080 \\
\hline “t”" calculado & 25,010 & 25,079 & 1,198 \\
\hline \multicolumn{4}{|l|}{ Soja } \\
\hline Parâmetro “a” & 102,971 & 111,821 & 32,350 \\
\hline Parâmetro "b” & $-2,613$ & $-3,591$ & 0,576 \\
\hline "t" calculado & 21,371 & 35,216 & 4,426 \\
\hline \multicolumn{4}{|l|}{ Trigo } \\
\hline Parâmetro “a” & 100,217 & 106,621 & 22,734 \\
\hline Parâmetro "b” & $-2,880$ & $-3,558$ & 0,225 \\
\hline “t” calculado & 20,335 & 24,433 & 1,930 \\
\hline
\end{tabular}

Fonte: Cálculos dos autores a partir de dados primários da EMATER-RS.

$t$ tabelado para 40 graus de liberdade, em razão do intervalo da tabela passar diretamente de 30 para 40 .

milho, trigo e arroz não apresentaram qualquer tendência, sendo que os produtos soja e feijão apresentaram, respectivamente, uma leve tendência de alta e de queda de preços.

No que diz respeito aos produtos aqui analisados, os resultados confirmam as conclusões de SOUZA et al. (2006) para os preços da pecuária, bem como de FARINA \& NUNES (2004), segundo as quais os produtos do setor primário, ao contrário de terem sustentado a estabilização monetária após o Plano Real, beneficiaram-se dela, pela redução, e mesmo reversão em alguns casos, da tendência histórica de declínio de seus preços.

\section{CONCLUSÕES}

O presente trabalho demonstra que todos os produtos agrícolas analisados apresentaram desvalorização significativa em seus preços reais de 1973 a 2005.

Constatou-se que a década de 80 foi o período de maior declínio de preços, sendo observado este comportamento de queda para todos os produtos analisados. O período pós-Plano Real foi marcado pela inexistência de tendência de preços para arroz, trigo e milho, sobretudo pela forma errática com que os mesmos variaram, e pela tendência de elevação de preços da soja e de declínio de preços do feijão.

\section{REFERÊNCIAS}

BARROS, J.R.M. et al. Os efeitos da pesquisa agrícola para o consumidor. São Paulo: EMBRAPA - FIPE, 2001. 66p. (Relatório final de pesquisa).

CARMO, M.S. (Re)estruturação do sistema agroalimentar no Brasil: a diversificação da demanda e a flexibilização da oferta. São Paulo : IEA, 1996. 255p. (Coleção Estudos Agrícolas, 5).

FARINA, E.M.M.Q; NUNES, R. Para além da agricultura: o efeito "treadmill" no sistema agroindustrial de alimentos no Brasil. Revista Economia Aplicada, São Paulo, v.8, n.2, p.348-376, 2004.

FINAMORE, E.B.M.C.; GOMES A.P. Uma alternativa à análise de tendência em séries temporais. In: CONGRESSO BRASILEIRO DE ECONOMIA E SOCIOLOGIA RURAL, 37., 1999, Foz do Iguaçu, PR. Anais... Brasília: SOBER, 1999. p.1-9.

Ciência Rural, v.37, n.4, jul-ago, 2007. 
GASQUES, J.G.; CONCEIÇÃO, J.C.P.R. Transformações estruturais da agricultura e produtividade total dos fatores. Brasília: IPEA, 2000. 60p. (Texto para Discussão n. 768).

GRAZIANO DA SILVA, J. A nova dinâmica da agricultura. Campinas: UNICAMP, 1998. 211p.

JOHNSTON, J. Métodos econométricos. São Paulo: Atlas, 1977. 313p

KARMEL, P.H.; POLASEK, M. Estatística geral e aplicada à economia. 2.ed. São Paulo: Atlas, 1976. 601p.

KAZMIER, L.J. Estatística aplicada à economia e administração. São Paulo: McGraw-Hill, 1982. 376p.
REZENDE, G.C. Estado, macroeconomia e agricultura no Brasil. Porto Alegre: UFRGS, 2003. 246p.

SOUZA, R.S. et al. Tendência histórica de preços pagos ao produtor na pecuária do Rio Grande do Sul, Brasil. Ciência Rural, Santa Maria - RS, v.36, n.5, p.1511-1517, 2006.

STEVENSON, W.J. Estatística aplicada à administração. São Paulo: Harper \& Row do Brasil, 1981. 490p.

VEIGA, J.E. Uma linha estratégica de desenvolvimento agrícola. In: ENCONTRO NACIONAL DE PÓS-GRADUAÇÃO EM ECONOMIA, 18., 1990, Brasília, DF. Anais... Brasília: ANPEC, 1990. p.987-1009. 